Repenser l'extraversion

Des usages de l'empowerment, du genre et du féminisme dans les associations de femmes bédouines du Néguev (Israël)

To rethink extraversion: Uses of empowerment, gender and feminism in Bedouin women's organizations in the Neguev (Israel)

\title{
Elisabeth Marteu
}

\section{(2) OpenEdition \\ Journals}

Édition électronique

URL : http://journals.openedition.org/conflits/18192

DOI : $10.4000 /$ conflits. 18192

ISSN : $1777-5345$

Éditeur :

CCLS - Centre d'études sur les conflits lilberté et sécurité, L'Harmattan

Édition imprimée

Date de publication : 30 décembre 2011

Pagination : $57-77$

ISBN : 978-2-296-55700-0

ISSN : 1157-996X

Référence électronique

Elisabeth Marteu, «Des usages de l'empowerment, du genre et du féminisme dans les associations de femmes bédouines du Néguev (Israël) », Cultures \& Conflits [En ligne], 83 | Automne 2011, mis en ligne le 04 janvier 2013, consulté le 30 mars 2021. URL : http://journals.openedition.org/conflits/18192 ; DOI : https://doi.org/10.4000/conflits.18192 


\section{Repenser l'extraversion}

Des usages de l'empowerment, du genre et du féminisme dans les associations de femmes bédouines du Néguev (Israël)

\section{Elisabeth MARTEU}

Elisabeth Marteu est docteure en science politique, chargée de cours à l'Institut d'Études Politiques de Paris. Spécialisée sur le conflit israélo-palestinien et les questions de genre au Moyen-Orient, elle a soutenu en 2009 une thèse sur les associations de femmes arabes palestiniennes en Israël. Elle poursuit aujourd'hui des recherches sur les mobilisations féminines/féministes transfrontalières, les rapports entre genre et nation dans un contexte globalisé.

Dléthore de travaux s'attachent depuis une quinzaine d'années à décons1 truire les visées démocratisantes et lénifiantes des financements internationaux à destination des sociétés du Sud, en particulier à destination des femmes (voir l'introduction dans ce numéro). Il ne fait plus aucun doute aujourd'hui que le foisonnement associatif féminin dans les années quatre-vingt-dix dans les pays du Sud est en partie lié à cette nouvelle manne financière et à ces ressources organisationnelles ayant participé de la professionnalisation des associations de femmes. Cette ressource matérielle a eu des effets immédiats sur les répertoires d'action de ces associations. Elle a pu provoquer des phénomènes de concurrence, de fragmentation et des tensions sociales et politiques dans des pays où les structures de femmes ont longtemps été des actrices essentielles dans les activités de bienfaisance et les mouvements de libération nationale. Elle a également accentué la dépendance financière à l'égard des bailleurs de fonds internationaux. La condition des femmes dans le monde arabe a clairement été identifiée depuis les années quatre-vingt-dix comme un indicateur de développement, de démocratisation et de pacification par les organisations internationales (ONU, FMI, BM) et les bailleurs de fonds. Cette quête internationale de démocratisation dans cette région du monde, avant tout pour des raisons économiques et sécuritaires, a donc accompagné l'intérêt des organisations internationales pour le soutien des forces réformistes, en particulier des femmes. Dans le contexte israélo-palestinien où les relations internationales et transnationales ont été au cœur des processus internes des sociétés politiques, 
les rouages de l'extraversion ne peuvent donc faire l'économie d'une contextualisation régionale et internationale ${ }^{1}$.

L'objectif de cet article est d'analyser l'usage de l'extraversion dans l'action collective ${ }^{2}$ des femmes, en étudiant plus particulièrement la manière dont les notions de "genre ", de «féminisme » et d' « empowerment ${ }^{3}$ " sont développées, représentées et redéfinies en local par les associations de femmes bédouines du Néguev en Israël. À partir d'une réflexion sur la circulation de ces notions, leur manipulation et leur usage en local, il s'agira d'interroger la manière dont les associations de femmes se rapproprient les termes de l'aide internationale pour construire (non sans tensions et difficultés) leurs propres discours et programmes d'action. C'est précisément dans cette approche multi-niveau que cette recherche porte sur la position singulière des associations bédouines du Néguev, minorité de la minorité arabe palestinienne en Israël.

Les Bédouins du Néguev constituent aujourd'hui une communauté d'environ 170000 personnes, musulmanes, considérées comme partie intégrante de la minorité arabe palestinienne en Israël (elle même représentant environ $18 \%$ de la population israélienne). À la suite de la première guerre israéloarabe (1947-1949), ils se retrouvèrent sur le territoire israélien et acquirent la citoyenneté israélienne au début des années cinquante. Déjà partiellement sédentarisés, les Bédouins du Néguev connurent à partir des années soixantedix un processus d'urbanisation forcée sous l'impulsion des autorités israéliennes. Si la moitié d'entre eux continue à vivre éparpillée dans le désert du Néguev dans des conditions socioéconomiques d'extrême précarité, l'autre moitié vit aujourd'hui dans des villes et villages récemment construits. L'urbanisation des Bédouins eut des effets immédiats sur le statut des femmes ${ }^{4}$ et la mobilité de la frontière privé/public, d'abord en reconfigurant leur fonction sociale (elles perdirent certaines de leurs prérogatives dans la construction de la tente et le pâturage des chèvres), ensuite en créant une

1. Sur la notion d'extraversion, au sens d'ouverture sur l'extérieur, cet article s'inscrit dans la continuité des travaux de Jean-François Bayart sur l'Afrique, qui a montré combien il fallait « dépasser la distinction stérile entre la dimension interne des sociétés africaines et leur insertion dans le système international [...] Le rapport de l'Afrique au reste du monde n'est pas d'ordre relationnel. Il ne relève pas de l'extranéité. Il est au contraire consubstantiel à sa trajectoire historique » (Bayart J.-F., «L'Afrique dans le monde : une histoire d'extraversion », Critique internationale, $\mathrm{n}^{\circ} 5,1999$, pp. 105).

2. Sur l'extraversion militante, voir notamment les travaux de Pommerolle M.E., Siméant J. (eds), Un autre monde à Nairobi - Le Forum social mondial 2007 entre extraversions et causes africaines, Paris, Karthala, 2008.

3. L'empowerment peut se traduire par la responsabilisation, la prise en charge de soi-même, la capacité d'action ou encore le pouvoir d'agir. Sur cette question, voir également l'article d'Isabelle Guérin dans ce numéro.

4. Jakubowska L., Urban Bedouin, Social Change in a Settled Environment, Albany, State University of New York, 1985. Lewando-Hundt G., Women's Power and Settlement: the Effect of Settlement on the Position of Negev Bedouin Women, MA Thesis, University of Edinburg, 1978. 
proximité entre des voisines bédouines découvrant leur nouveau statut de femmes au foyer, enfin en réinventant les contraintes et les règles d'honneur pesant sur les femmes. C'est précisément dans ces nouveaux centres urbains (particulièrement pauvres) que se sont développées au début des années quatre-vingt-dix des associations de femmes souvent dirigées par des jeunes femmes éduquées et actives, issues de familles ou de lignages dominants 5 .

À la différence des autres régions d'Israël/Palestine, et surtout des autres populations palestiniennes, les Bédouines ont tardivement connu l'expérience associative. Les Palestiniennes des grands centres urbains, comme Jérusalem, Nazareth, Acre, Naplouse ou Bethleem, avaient établi des structures féminines dès le début du XXe siècle ${ }^{6}$. Ces associations, d'abord charitables et sociales, servirent ensuite de lieu de mobilisation contre les autorités britanniques et les implantations sionistes, jusqu'en 1948. Très tôt, les Palestiniennes se sont donc mobilisées sur le double registre de l'identité féminine (pas encore formulée en termes féministes) et de l'identité nationale arabe palestinienne. La mobilisation féminine palestinienne jouit donc aujourd'hui d'un héritage ancien et endogène, ayant fortement marqué les modes d'action collective des femmes tant en Israël/Palestine qu'au sein des populations palestiniennes réfugiées dans les autres pays du Proche-Orient. La communauté bédouine ne bénéficie pas de cette expérience tant philanthropique que militante. Les Bédouines ne commencèrent à se mobiliser dans des structures collectives que dans les années quatre-vingt-dix, précisément au moment de l'arrivée des bailleurs internationaux, du succès des programmes " genre et développement » et des transformations de l'associatif en nouveau marché du travail. Cette singularité spatio-temporelle a donc joué sur le développement de leurs modes d'action et sur la manière dont l'épistémologie féministe a pu être appréhendée. Trois associations feront l'objet ici d'une attention particulière : d'abord Nisa al Laqiyya (les femmes de Laqiyya), créée en 1991 et qui produit des broderies bédouines, ensuite Sidreh, créée officiellement en 1998 et qui fonctionne autour d'une fabrique de tapis, et enfin $M a^{\prime} a n$, créée plus récemment dans les années deux-mille, qui sert de forum pour les associations de femmes bédouines du Néguev.

Comme les membres de l'association Nisa al Laqiyya aiment le rappeler : «l'association remplace le puits, le point d'eau et de rencontre pour toutes les

5. La communauté bédouine du Néguev était répartie en trois groupes sociaux : les Bédouins possédants, les paysans (Fellahin) qui louaient les terres aux Bédouins pour les exploiter, et les anciens esclaves dits Humran (rouge) originaires de pays africains comme le Soudan. Si cette organisation hiérarchique est moins prégnante aujourd'hui, du fait des récents bouleversements économiques et matrimoniaux, elle demeure cependant inscrite dans les mémoires et fait encore sens dans certaines relations sociales (Meir A., As Nomadism Ends, Boulder, Lynne Rienner Publishers, 1997 ; Parizot C., Le mois de la bienvenue. Réappropriations de mécanismes électoraux et réajustements de rapports de pouvoir chez les Bédouins du Néguev (Israël), Thèse de Doctorat, Paris, EHESS, 2001).

6. Fleischmann E., The Nation and Its New Women: the Palestinian Women's Movement, 19201948, Berkeley, Los Angeles, London, University of California Press, 2003. 
femmes des environs 7 ». En effet, si les associations représentent avant tout de nouveaux espaces de socialisation, de distraction et de dialogue, elles sont aussi devenues des structures professionnelles salariant une partie de leurs membres et dépendantes exclusivement d'aides extérieures. Cette réalité rend compte de la forte présence des organisations internationales dans le travail des associations locales de femmes, y compris dans les régions les plus isolées et marginalisées du Proche-Orient. Le contexte des relations entre associations de femmes bédouines et bailleurs internationaux est d'autant plus singulier qu'elles se situent dans le cadre de l'État israélien. Non seulement les Bédouins restent traités comme des citoyens de seconde zone, mais ils revendiquent de plus en plus leur pleine appartenance au peuple palestinien tout en soutenant massivement les partis islamiques. Pour autant, y compris au sein de la population arabe palestinienne d'Israël, les Bédouins sont jugés plus conservateurs et traditionalistes, notamment sur les questions de genre. C'est pourquoi les femmes, souvent considérées comme des agents potentiels de changement social, reçoivent l'attention des autorités israéliennes et des organisations internationales. Elles se retrouvent ainsi à la coextension de multiples rapports de pouvoir qui conditionnent leurs modes d'action associative.

Se pose ainsi la question de la nature et des enjeux de cette négociation multi-niveau, en particulier dans la manière dont ces associations font usage des concepts de "genre », de "féminisme » et d' « empowerment». Aussi paraît-il particulièrement intéressant d'éclairer les rouages de l'extraversion, en se concentrant sur l'usage de ces notions qui ont pu être considérées comme centrales dans les mobilisations de femmes, mais qui font aussi l'objet de débats au point de susciter des refus, des aménagements et des redéfinitions. La force de l'intersectionnalité ${ }^{8}$ ou de la coextensivité/consubstantialité des rapports de pouvoir ${ }^{9}$ est aussi déterminante pour comprendre comment les Bédouines construisent leurs répertoires d'action en articulant une identité de femmes, bédouines, arabes, musulmanes, palestiniennes, israéliennes. Ainsi, si l'empowerment fait aujourd'hui l'objet d'un très large usage et affichage de la part des bailleurs comme des associations, il en va en revanche différemment du concept de genre, pas toujours utilisé dans son sens subversif originel, et encore moins du féminisme qui reste connoté de visées hégémoniques néocoloniales occidentales. L'intérêt est donc ici de comprendre comment s'opèrent les choix dans l'usage et la reformulation de ces notions sous l'effet de ressources et de contraintes multi-niveaux (locales, nationales et internationales).

7. Entretiens et observation participante réalisés dans le cadre de ma thèse de science politique soutenue à l'IEP de Paris (en cotutelle avec l'Université Ben Gourion d'Israël) en mars 2009. Les associations de femmes arabes d'Israël. Actrices et enjeux de nouvelles formes de mobilisation palestinienne en Israël.

8. Crenshaw K., "Mapping the Margins: Intersectionality, Identity Politics, and Violence against Women of Color”, Stanford Law Review, vol. 43, n6, 1991, pp. 1241-1299. 


\section{L'entreprise d'empowerment des Bédouines du Néguev}

Origine et contenu des activités d'empowerment

Dans le village bédouin de Laqiyya (établi dans les années quatrevingts/quatre-vingt-dix et comptant environ 9000 habitants), les activités associatives sont très limitées. Hormis une association culturelle et sportive créée dans les années quatre-vingts (qui ferma rapidement ses portes), la première association de femmes, Nisa al Laqiyya (les femmes de Laqiyya) fut établie au début des années quatre-vingt-dix.

Cette association fut créée par les femmes de la famille As Sana' (un des lignages dominants du village), en particulier par Amal, âgée à l'époque d'une vingtaine d'années. Etudiante à l'université Ben Gourion, Amal (aujourd'hui directrice d'une association de coexistence judéo-arabe) exprima très jeune le souhait d'aider les femmes de sa famille, notamment par des cours d'alphabétisation. Le premier programme mis en place en 1992 fut celui des «broderies du désert » (tatriz al badiya). L'objectif était alors de se servir du savoir-faire local des femmes pour développer des activités économiques. Ces activités de broderie font travailler aujourd'hui quelques soixante-dix Bédouines du village, originaires de familles et lignages différents. Si les dirigeantes restent exclusivement liées à la famille As Sana' (directement ou par le jeu des anciennes solidarités et allégeances tribales), en revanche les bénéficiaires viennent de divers groupes sociaux. Toutes ne reçoivent pas assez d'argent à la fin du mois pour réclamer leur indépendance financière, les revenus pouvant aller de quelques dizaines à quelques centaines d'euros. Cependant, plus que l'enrichissement, c'est avant tout la rétribution matérielle d'un travail reconnu et spécialisé qui est important. Autonomisation économique et préservation d'un artisanat local s'articulent dans un même souci d'empowerment pour les femmes. Alors que le taux d'activité des Bédouines dans les villes planifiées est de seulement $13 \%{ }^{10}$, ces associations entendent promouvoir, à petite ou moyenne échelle, le travail salarié des femmes et leur autonomisation personnelle.

Lorsque les brodeuses se rendent à l'association toutes les semaines pour ramener leurs broderies (commandées à l'avance suivant des patrons définis par les dirigeantes), elles en profitent pour passer du temps à discuter avec les autres femmes présentes. Une fois par mois en moyenne, le jour de paie, elles sont invitées à l'association pour assister à une conférence sur des thèmes divers comme la santé, les conseils diététiques, les produits cosmétiques, ou

9. Kergoat D., « Division sexuelle du travail et rapports sociaux de sexe », in Hirata H., Laborie F., Le Doaré H., Senotier D. (ed.), Dictionnaire critique du féminisme, Paris, PUF, 2000, pp.35-44.

10. Working Group on the Status of Palestinian Women in Israel, CEDAW Alternative Report, 2005, p.41. 
encore l'éducation des femmes et des enfants. Ces rencontres ont pour but de sensibiliser les femmes à des sujets sociaux, mais parfois également de les conscientiser sur leurs droits, notamment dans le cadre de discussions sur les violences domestiques. Ces sujets restent néanmoins très rarement abordés, les dirigeantes de l'association privilégiant un investissement sur des questions économiques, sociales et sanitaires considérées comme prioritaires.

Nisa al Laqiyya se situe dans un local familial autour d'une grande tente bédouine, reconstituée spécialement pour les activités de l'association. Cette tente permet de recevoir des touristes (le plus souvent israéliens) et les bailleurs de fonds, pour leur présenter l'association. Très régulièrement, $\mathrm{Na}$ 'ama, sœur ainée d'Amal et actuelle dirigeante de l'association, revêt sa robe traditionnelle bédouine, son sheish blanc (voile), sert le café et le thé à ses hôtes, voire parfois le traditionnel magluba (plat palestinien). Elle leur présente alors en hébreu, en arabe (ou en anglais aidée d'un traducteur) le fonctionnement de l'association. Elle présente les différents projets de l'association, en priorité le projet «broderies». Les broderies bédouines ont une signification sociale. Selon les motifs et les couleurs, il était autrefois possible d'identifier le statut des femmes qui les portaient. Ces broderies étaient cousues sur les robes (thob) à fond noir, essentiellement un large plastron sur la poitrine et quelques bandes sur les côtés et le bas. Des symboles végétaux, de même que des relations sociales (relations belles mères-brus) y étaient apposés, le plus souvent en rouge pour les femmes mariées et en bleu ou vert pour les jeunes filles. Ces impressions ont été réutilisées à des fins marchandes par l'association pour en faire des sacs, des châles, et des objets de décoration d'intérieur. De nouveaux dessins et symboles ont même été inventés comme la reconfiguration tribale et la perte de pouvoir des cheikhs. Les trois autres projets présentés sont ceux de la garderie pour enfants, située non loin de l'association, de la bibliothèque ambulante, qui circule dans quelques villages bédouins en proposant des livres pour enfants, et du programme leadership, qui vise à soutenir l'engagement de jeunes bédouines dans leur communauté (cours d’alphabétisation, bénévolat, services, etc.).

\section{Empowerment: débats autour d'une traduction linguistique et pratique}

Ces trois projets sont caractéristiques des priorités actuelles des associations de femmes bédouines : travail, éducation et responsabilisation des jeunes femmes. Quand $\mathrm{Na}$ 'ama fait cette présentation, elle plaide pour une amélioration du statut de la femme arabe bédouine (tabsin makan al mar'a al 'arabiyya al badawiyya) ou l'empowerment (tamkin) mais dans le respect de la « routine quotidienne des femmes, sans bousculer leur mode de vie traditionnel et les valeurs familiales $11 »$. Lorsqu'il s'agit de traduire ses propos en anglais,

11. Ce principe est souvent rappelé par les dirigeantes et se trouve d'ailleurs écrit sur leur site internet. http://www.desert-embroidery.org. Consulté le 15 avril 2011. 
notamment devant les représentants des bailleurs de fonds, c'est immédiatement l'expression «women's empowerment » qui est employée.

Elle correspond d'ailleurs aux propres lignes de financement des bailleurs, même s'ils n'utilisent pas tous le terme 12. L'association recevait en 2007 des aides du New Israel Fund, du Global Fund for Women et de la fondation Heinrich Böll. Le New Israel Fund finance des associations de femmes arabes à travers les programmes d'aide spécifiques «femmes » et «Palestiniens israéliens 13 ». Le Global Fund for Women finance des projets axés sur "l'égalité et les droits des femmes 14 ». Heinrich Böll finance, entre autres, des projets sur la « démocratie » et le «genre 15 ». Cependant, la vente de broderies ne suffit pas pour financer les autres activités. Régulièrement la production est réduite à deux ou trois jours dans la semaine pour s'adapter à la baisse de la demande. Le principal reproche qui a été fait à l'association, notamment par la fondation Heinrich Böll, réside dans son manque de professionnalisme, sa gestion trop familiale et l'absence d'objectif précis au regard de la condition des femmes ${ }^{16}$. Nisa al Laqiyya reste ce que les bailleurs appellent des "community based organizations » ou des "grassroots organizations » pour signifier leur ancrage local et populaire et leur faiblesse organisationnelle. L'association a essayé de transformer ses modes d'action, en créant un site internet, en recrutant en dehors de la famille As Sana' ou encore en élaborant un discours plus élaboré sur l'empowerment et la responsabilisation féminine individuelle. Mais elle reste profondément ancrée dans une réalité difficile où les femmes veulent d'abord entendre parler de ressources économiques et d'éducation avant de revendiquer des droits.

Là est justement la problématique des financements internationaux qui cherchent d'une part à promouvoir l'empowerment à partir d'associations

12. L’Union européenne, très présente en Israël/Palestine, finance des associations dans le cadre de l'Instrument européen pour la démocratie et les droits de l'homme. Parmi les objectifs de l'IEDDH figure clairement la lutte contre les violences faites aux femmes et leur empowerment pour la représentation et participation politique. ht t p:// ec.europa.eu/europeaid/what/human-rights/documents/ eidhr_strategy_paper_2011_2013_com_decision_21_april_2011_text_published_on_ internet_en.pdf

13. Le New Israel Fund est une organisation fondée par une famille de philanthropes juifs américains finançant depuis les années quatre-vingts les associations israéliennes, y compris arabes.

14. Le Global Fund for Women a été créé dans les années quatre-vingts pour venir en aide aux associations de femmes dans le monde, dans le but de promouvoir les droits des femmes, le changement social, la paix et l'égalité. Cf. http://www.globalfundforwomen.org/who-weare/our-mission. Consulté le 10 mai 2011.

15. L’organisation Heinrich Böll est affiliée au parti écologiste allemand et finance des projets axés sur l'écologie, la démocratie, les droits de l'homme, l'auto-détermination et la justice, avec une attention particulière portée aux questions de genre. http://www.boell.org/web/137.html. Consulté le 10 mai 2011.

16. Réunion-bilan à l'issue des trois années de financement par la fondation Heinrich Böll 20032006, Haïf, 2006. Pour une analyse plus large sur la prégnance du patriarcat et les difficultés que cela pose aux acteurs du développement voir l'article de Suad Joseph "Patriarchy and Development in the Arab World", Gender and Development, vol 4, n², Juin 1996, pp. 1419. 
locales proches des besoins élémentaires des femmes, et d'autre part à assurer la rentabilité de leurs financements. Une des critiques majeures faite à l'usage de l'empowerment par les organisations internationales réside justement dans leur focalisation sur une approche individuelle de la prise en charge (notamment économique) des femmes, dans une perspective du développement liée à la lutte contre la pauvreté, mais qui ne remet pas en cause les rapports de pouvoir et de domination entre les hommes et les femmes. Le terme d'empowerment perd ainsi toute sa dimension critique et s'éloigne de l'idée de " pouvoir » élaborée par les mouvements féministes. L'empowerment se limite alors à la capacité d'action sans formuler de revendications profondes remettant en cause les rapports hommes-femmes ${ }^{17}$, et devenant ainsi une version «individualiste, dépolitisée, verticale et instrumentale 18 ».

Par ailleurs, l'empowerment est très difficilement évaluable, tant par des méthodes quantitatives (comme les indicateurs sexo-spécifiques) que qualitatives. La notion de pouvoir, élaborée par les organisations féministes et reprenant les réflexions de Foucault, identifie quatre types de pouvoir : le " pouvoir sur » (domination, subordination, résistances), le «pouvoir de ( capacité de prendre des décisions et d'accomplir des choses), le « pouvoir avec » (solidarité, mobilisations collectives), le « pouvoir intérieur » (estime de soi, changements pour soi) ${ }^{19}$. L'empowerment vise donc bien à l'acquisition de pouvoir (en priorité ici le «pouvoir de », le "pouvoir intérieur » et le " pouvoir avec») et au libre choix individuel pour décider de sa vie. Ce qui impose de prendre en considération les situations de vulnérabilité, de contrainte et de domination qui conditionnent la décision et le choix individuel. Des associations comme Nisa al Laqiyya sont conscientes des contraintes sociales, économiques, culturelles qui pèsent sur les femmes bédouines et soutiennent ainsi la responsabilisation individuelle par le travail et les ressources économiques. Elles soutiennent le développement personnel des femmes, l'estime, la confiance en soi et pensent, à juste titre, que les femmes bédouines ont besoin de se sentir intégrées à la société et à la vie collective pour exister pleinement.

L'empowerment tel qu'il est utilisé par Nisa al Laqiyya s'apparente à une amélioration du statut et des conditions de vie des femmes. On peut y déceler un fond critique sur la marginalisation des femmes, leur isolement, leur fragilité face à des conditions socioéconomiques difficiles (pauvreté, chômage, analphabétisme, dépendance, etc.). Mais il ne porte pas publiquement une cri-

17. Mestrum F., «L'“empowerment”, le pouvoir et la capacité d'action », Chronique féministe, 2000 , pp. $70-75$.

18. Calvès A.E., " “Empowerment” : généalogie d'un concept clé du discours contemporain sur le développement ", Les mots du développement. Trajectoires et pouvoirs, Revue Tiers Monde, 4, 2009, pp. 735-749.

19. Pour une étude détaillée voir l'article de Charlier S., «L'analyse de l'empowerment des femmes qui participent à une organisation de commerce équitable. Une proposition méthodologique ", in Auroi C., Yepez I., Économie Solidaire et commerce équitable. Acteurs et actrices d'Europe et d'Amérique latine, Presses Universitaires UCL/IUED, 2006. 
tique de l'inégalité hommes-femmes et n'est pas l'équivalent d'une "émancipation ", au sens d'affranchissement de toute forme de rapports dominants. Outre les rapports de genre, la question de l'empowerment des femmes bédouines doit se penser dans le contexte singulier d'une minorité arabe palestinienne au sein de l'État israélien. Or, les programmes d'empowerment financés par les bailleurs internationaux occultent largement cette problématique. Ils ciblent une « minorité ethnique pauvre », mais ne considèrent pas la dimension nationale politique liée au conflit israélo-palestinien et à la marginalisation des Bédouins, considérés comme des citoyens de seconde zone, voire une minorité dangereuse en Israël. Comme le souligne Eileen Kuttab, dans le contexte palestinien, les associations de femmes doivent comprendre l'empowerment des femmes (individuel et collectif) dans le cadre plus large d'une lutte nationale et d'une lutte pour l'indépendance sociale et économique 20. Sans cette articulation des luttes, c'est l'expérience même des femmes palestiniennes qui serait décontextualisée et donc dépolitisée ${ }^{21}$. La coextensivité et consubtantialité des rapports de pouvoir rend inconséquente toute action d'émancipation féminine déconnectée des contraintes socioéconomiques et politiques nationales. En outre, au regard de l'importance socioéconomique et politique du fonctionnement communautaire (familial, ethnique, confessionnel, etc.) au Moyen-Orient, les femmes doivent négocier leurs relations collectives avec les autres communautés tout en négociant leurs relations en tant qu'individu (femme) dans le cadre national voire international ${ }^{22}$. Les Bédouines du Néguev sont donc plus que jamais confrontées à ce double registre de l'empowerment individuel et collectif (de genre, social et national) dans un contexte conflictuel, de fragmentation communautaire au sein d'un État à domination juive et de forte discrimination socioéconomique.

\section{Pouvoir des femmes et empowerment à l'aune des études féministe postcolo- niales}

L'empowerment des femmes fait partie des sujets de débat qui animent les études féministes en général et le féminisme postcolonial en particulier. Pléthore de travaux se sont développés depuis les années quatre-vingts pour repenser la place des femmes dans le monde arabe et musulman, et pour reformuler des luttes indépendamment d'une épistémologie féministe considérée comme occidentalo-centrée et hégémonique. Les féministes arabes ont souli-

20. Kuttab E., "Empowerment as Resistance: Conceptualizing Palestinian Women's Empowerment”, Development, 53(2), 2010, pp. 247-253.

21. Sur la thématique de la professionnalisation des associations de femmes palestiniennes, leur ONGisation et dépolitisation, voir entre autres les contributions dans Gender and Development, Working Paper 3, Institute of Women Studies, 1995 ; Hammami R., "NGOs: The Professionalization of Politics", Race and Class, 37(2), 1995, pp. 51-63; Jad I., "The NGO-isation of Arab Women's Movements", Al Raida, vol. XX, n¹00, Winter 2003, pp. 3747 ; Kuttab E., "Palestinian Women's organizations: Global Cooption and Local Contradiction”, Review of Women's Studies, n ${ }^{\circ}, 2009$, pp. 64-74.

22. Joseph S. "Gender and Citizenship in Middle Eastern States", Middle East Report, n¹98, 1996, pp. 4-10. 
gné d'une part que les rapports de pouvoir s'inscrivaient dans des contextes de domination coloniale et de lutte nationaliste, et d'autre part qu'il fallait se départir d'une vision orientaliste des femmes arabes et musulmanes comme victimes totales du patriarcat ${ }^{23}$. Des universitaires comme Lila Abu Lughod ont ainsi contribué à repenser les rapports de genre dans le monde arabe et notamment à revisiter le concept de "pouvoir » et de « résistance » à l'aune des pratiques des femmes ${ }^{24}$. Lila Abu Lughod s'est opposée à la vision totalisante des femmes arabes comme victimes de la domination masculine et a cherché à mettre au jour des formes de transaction. Elle s'inscrit ainsi dans la veine foucaldienne, en partant du postulat suivant : « là où il $\mathrm{y}$ a du pouvoir il y a de la résistance », en ajoutant alors « là où il y a de la résistance il y a du pouvoir ». Plus que la question de l'« agency » qu'elle accrédite d'une vision "romancisée de la résistance ", c'est la question des rapports de pouvoir négociés et reconfigurés qui l'intéresse. Pour cela, elle se base sur ses recherches menées auprès de la tribu bédouine égyptienne des Awlad 'Ali. Elle identifie différentes formes «traditionnelles » de résistance utilisées par les femmes, comme l'appropriation de la répartition des espaces sexués en maitrisant et protégeant l'espace des femmes (lieu des secrets, des interdits, etc.), les résistances et refus de mariages arrangés, ou encore les moqueries sur les hommes et la masculinité. Elle actualise son analyse en identifiant de nouvelles formes de résistance chez les Bédouines sédentarisées, comme le port de vêtements islamiques ou la consommation de masse, sujets à de nouvelles formes de restriction et de contrôle sur les femmes. La résistance serait ainsi un « diagnostic du pouvoir », les actes de résistance s'inscrivant dans le système de pouvoir et de contraintes en interagissant et évoluant avec lui.

L'analyse faite par Lila Abu Lughod s'adapte parfaitement au contexte bédouin du Néguev, où s'observe de la même manière une reconfiguration des formes de résistance et des rapports de pouvoir sous l'effet de l'urbanisation, de la sédentarisation, de la précarisation économique dans un système néolibéral, ou encore du renouveau des règles d'honneur. Dans ce contexte, les femmes sont plus que jamais des garantes de valeurs dites traditionnelles et authentiques. À l'heure où elles investissent plus massivement l'espace public israélien (notamment par l'éducation et le travail), elles connaissent aussi un resserrement de l'étau communautaire contrôlant leurs agissements et recomposant les rapports de genre, soit au nom d'une culture bédouine réinventée, soit au nom de l'Islam. L'espace public israélien est non seulement le lieu de la mixité ethnique et sexuelle, mais il est aussi le lieu de la domination économique et politique juive, où les Bédouins (et les citoyens arabes en général)

23. Abu Lughod L., "Orientalism and Middle East Feminist Studies", Feminist Studies, vol. 27, $\mathrm{n}^{\circ} 1,2001$, pp. 101-113. Sur le féminisme dit post- ou anti-colonial dans le monde arabe et musulman, voir les travaux de Fatima Mernissi, Leila Ahmed, Deniz Kandiyoti ou encore Margot Badran.

24. Abu Lughod L., "The Romance of Resistance: Tracing Transformations of Power Through Bedouin Women”, American Ethnologist, vol. 17, n¹, 1990, pp. 41-55. 
expérimentent au quotidien les rapports de domination. Les rapports de pouvoir de genre sont donc encastrés dans des rapports de pouvoir socioéconomiques et ethnico-confessionnels. Plus largement ici, le couple pouvoir/résistance s'inscrit dans la multidimensionnalité des rapports qu'entretiennent les femmes bédouines avec les hommes de leur communauté, l'État israélien ou encore les organisations internationales. Loin d'une vision romancisée des associations de femmes bédouines comme symbole d'une résistance au patriarcat, ces structures agissent dans le cadre de ressources et de contraintes, de rapports de pouvoir en permanente recomposition. Mais dans quelle mesure les associations de femmes peuvent-elles être des espaces de pouvoir et de capacité d'agir ? Les réponses sont multiples.

Les associations représentent d'abord de nouveaux espaces de socialisation et de dialogue. Elles sont ainsi un nouvel espace privé, féminin et sécurisé permettant la discussion, les confidences et les échanges sur des questions intimes, notamment sur les relations hommes-femmes. Mais elles sont aussi de nouveaux espaces publics où les femmes bédouines peuvent côtoyer des touristes, des visiteurs et des représentants israéliens ou étrangers, comme les représentants des bailleurs de fonds. Elles rencontrent ainsi des hommes indépendamment de leurs maris, fils ou frères. Alors que les contacts entre hommes et femmes bédouins restent encore très contrôlés et codifiés, les structures professionnelles représentent de nouveaux espaces de mixité ponctuelle. Il est d'ailleurs intéressant d'observer combien elles changent d'attitude avec des hommes étrangers ou israéliens juifs, se permettant de discuter, de plaisanter, alors qu'elles ne le feraient pas avec des hommes bédouins extérieurs à leur famille. Je me souviens ainsi de la visite d'un touriste français à l'association en avril 2005. Alors qu'il était en train d'échanger quelques mots avec une Bédouine, cette dernière prit ses distances et remit son voile sur une partie de son visage lorsqu'elle aperçut un homme du village dans les alentours. L'association est également un lieu de rencontre avec des interlocuteurs qui veulent les connaître et les aider, et un lieu où se reconfigurent les rapports dominants-dominés, notamment dans leurs relations avec les Juifs israéliens. Ces associations sont donc avant tout un lieu circonscrit de recomposition et de redéfinition des relations sociales et des rapports de pouvoir.

Ensuite, ces associations mettent au jour des formes d'autonomie et d'action pour les femmes qui, ne serait-ce que quelques heures par semaine, ont le sentiment de travailler pour elles et d'agir sans médiation masculine. Ces rétributions symboliques sont d'autant plus évidentes dans le cas des jeunes femmes éduquées qui trouvent dans l'associatif un nouveau marché du travail valorisant. Enfin, ces associations ont un rôle de représentation symbolique de l'émancipation féminine dans un contexte bédouin encore largement marqué par les rapports de pouvoir entre hommes et femmes. Ainsi, les deux associations du village de Laqiyya ont fait l'objet d'actes de vandalisme au cours des dix dernières années. L'association Sidreh a été cambriolée et obligée de 
déménager. L'association Nisa al Laqiyya a été victime d'un incendie criminel en 2005. Sans sur-interpréter les raisons de ces attaques, qui sont aussi probablement liées à des règlements de compte internes au village, il est certain que les membres des associations les ont en partie vécus comme une violence sexiste. Quelques jours après l'incendie, une manifestation fut organisée par des associations de femmes arabes en Israël pour marquer leur solidarité avec Nisa al Laqiyya et rappeler que malgré les actes d'intimidation (de la part des hommes), les femmes continueraient de se mobiliser pour leurs droits. C'est d'ailleurs un discours quasi similaire qui fut porté à la Commission sur le statut des femmes de la Knesset (parlement israélien) quelques semaines plus tard, lorsque les représentantes de l'association demandèrent une aide financière pour la reconstruction de leur local associatif. Leur discours fut d'autant plus apprécié par les membres de la commission, en particulier sa directrice de l'époque, députée juive du Likud (parti de droite), qu'il nourrissait les représentations collectives sur le conservatisme et la violence patriarcale de la communauté bédouine.

Si les travaux de Lila Abu Lughod sont passionnants pour comprendre les rouages complexes et conflictuels des processus d'émancipation individuelle, ils ne peuvent expliquer complètement les ressorts de la mobilisation collective des femmes dans l'espace associatif. Il faut ici faire une différence essentielle entre l'analyse de l'émancipation (individuelle) et l'analyse de l'action collective (en particulier du féminisme). L'étude des associations de femmes bédouines présente justement la singularité de se situer au croisement de ces deux dynamiques. Il est souvent plus aisé de réfléchir à l'émancipation individuelle dans un contexte où le féminisme est rejeté comme cadre discursif et normatif hégémonique colonial. Ces réticences, de la part des actrices comme de nombreuses universitaires, a largement stigmatisé le féminisme comme d'essence forcément occidentale, ce qui a limité l'émergence d'un mouvement collectif de lutte.

\section{Genre et féminisme : résonances, dissonances et réinterprétations}

\section{Entre droits, égalité et genre}

Si l'empowerment est présent dans la plupart des programmes d'action des associations de femmes bédouines, il en va différemment en revanche du concept de «genre». Il existe sur ce point une distinction entre l'association Nisa al Laqiyya et l'association Sidreh (basée dans le même village), qui a été créée à la même époque par des jeunes femmes de la famille As Sana' avec le même objectif d'empowerment économique, mais ici par la production de tapis bédouins. Les deux associations insistent sur le savoir-faire des femmes et le besoin de les rendre autonomes. De la même manière dans les deux associations, c'est à partir de la sacralisation d'une prétendue tradition artisanale locale, typiquement féminine, que se pense l'empowerment des femmes. En 
identifiant des "pratiques ancestrales féminines », elles réinventent leur singularité culturelle en reproduisant une division sexuelle du travail et donc leur besoin de penser l'émancipation dans le cadre des contraintes de la communauté bédouine.

Pour autant, Sidreh a mieux développé ses activités, d'abord en recevant plus de financements (notamment de la part d'Oxfam et de l'Union européenne), ensuite en mettant en place des programmes d'alphabétisation (dans le cadre d'un forum réunissant une dizaine de structures associatives locales), des cours d'entrepreneuriat local, et en créant des comités de femmes dans plusieurs villages reconnus et non reconnus du Néguev. Les dirigeantes de l'association précisent qu'elles cherchent à promouvoir « la participation, la représentation et les droits des femmes » et qu'elles essaient ainsi de travailler avec « les hommes et les institutions locales pour assurer leur accord et leur participation ». L'association espère ainsi pouvoir permettre aux femmes d'avoir des « outils pour intégrer le monde moderne en tant qu'individus indépendants ». En ce sens, Sidreh ancre ses activités dans une réflexion plus large sur les droits des femmes, les rapports hommes-femmes, sans affirmer clairement la domination masculine, ni celle des discriminations (tamyiz) ${ }^{25}$, mais en reconnaissant une marginalisation des femmes et une nécessaire négociation avec les hommes bédouins.

Les questions de genre sont ici partiellement abordées, puisque l'association ne se limite pas à promouvoir le travail des femmes, mais traite aussi directement des relations et des inégalités hommes-femmes. Pour autant, le concept de genre n'est pas utilisé dans le sens d'une remise en cause profonde des identités construites et des stéréotypes sexués, et il n'apparaît pas littéralement dans les programmes d'action de l'association. Ce qui n'empêche pas les dirigeantes de manipuler ce concept à l'oral lors des entretiens avec des bailleurs ou des universitaires. Elles savent qu'il fait sens et qu'il renvoie à un professionnalisme et une maitrise des termes en circulation dans l'espace des mobilisations de femmes. Par conséquent, elles se réserveront le choix de l'employer uniquement dans des espaces où il est légitime, par exemple dans leurs relations avec les organisations internationales.

Si certaines jeunes femmes manipulent le "genre ", c'est aussi parce qu'elles ont été sensibilisées à cette épistémologie féministe. Elles sont plutôt jeunes (la trentaine), maîtrisant l'anglais, sont diplômées de l'université Ben Gourion (souvent en études sociales), sont en contact avec les bailleurs étrangers et participent aux rencontres internationales. Hanan, une des dirigeantes de l'association Sidreb récemment formée à l'approche de genre, représente bien cette génération de Bédouines éduquées désireuses de changer la situa-

25. Le terme «discriminations » est utilisé davantage par les associations de droits de l'homme pour condamner les inégalités entre Juifs et Arabes en Israël. 
tion des femmes dans le Néguev. En juillet 2005, elle fut envoyée à New York pour présenter le rapport alternatif des associations de femmes palestiniennes d'Israël 26 devant le comité CEDAW 27 des Nations unies. Ce deuxième rapport alternatif (le premier ayant été soumis en 2001) comportait un chapitre sur les Bédouines, conformément à l'article 14 de la convention CEDAW relatif aux femmes rurales. Hanan a donc porté la voix des Bédouines à l'ONU en répondant aux questions du comité CEDAW qui se montra particulièrement intéressé par le sort des Bédouines, mais elle a aussi acquis les outils du plaidoyer international en participant aux sessions de formation de l'International Women's Rights Action Watch Asia Pacific (IWRAW).

L'IWRAW, créée en 1985 et ayant un statut consultatif à l'Economic and Social Council des Nations Unies (ECOSOC), fait office de consultant en capacity building, en méthodes de plaidoyer et en dissémination des informations relatives à la défense des droits des femmes. Pendant plusieurs jours, au mois de juillet 2005, cette organisation a proposé des séminaires de travail et des séances de préparation aux associations se présentant devant le comité CEDAW. En collaboration avec le comité CEDAW, IWRAW est donc chargé de former et préparer les associations au travail de plaidoyer et de lobbying sur les questions de genre. Les membres du groupe de travail sur le statut des femmes palestiniennes citoyennes d'Israël, dont Hanan, ont reçu un classeur comportant différents documents explicatifs sur la CEDAW, sur le mode de fonctionnement du comité, sur le vocabulaire à connaître (égalité, non discrimination, obligation étatique), sur le rôle des ONG, sur la manière de rédiger puis de présenter le rapport alternatif, sur les mécanismes de monitoring et de suivi de l'application sur le terrain de la convention, sur les arguments à opposer aux détracteurs de la convention, ainsi que des exercices pratiques d'élocution et d'argumentation sur l'égalité des sexes. Les membres de l'IWRAW leur ont également appris comment aborder de façon informelle les membres du comité CEDAW, notamment entre deux réunions dans les couloirs des Nations unies. Les membres du groupe de travail ont ainsi acquis les codes propres au plaidoyer international, la rhétorique, les méthodes de travail et surtout les outils de communication nécessaires. Par ailleurs, ces rencontres leur ont aussi permis d'établir un contact avec d'autres associations du Sud, d'échanger des expériences et des réflexions et d'intégrer ainsi le réseau féministe onusien.

26. Ce rapport alternatif fut rédigé par le groupe de travail sur le statut des femmes palestiniennes d'Israël qui regroupait les associations suivantes : Al Tufula, Women against Violence, Kayan, Ma'an, Arab Association for Human Rights (HRA) et Adalah.

27. Israël a ratifié la convention CEDAW - Convention on the Elimination of all forms of discrimination against Women - en 1991. Israël a émis des réserves sur l'application du paragraphe b de l'article 7 et sur l'article 16, qui ont trait à la participation des femmes aux postes de décision, à savoir plus précisément l'impossibilité pour les femmes d'exercer les fonctions de juges des tribunaux religieux, ainsi qu'à l'application de la législation religieuse sur le statut personnel. 
Hanan a donc été sensibilisée aux questions de genre, sous l'angle des droits et de l'égalité femmes-hommes. Cette formation ne l'a pas tant éveillée aux rapports de genre (dont elle a pertinemment conscience), mais elle a permis de mettre des mots, de conceptualiser les inégalités de genre et de mutualiser les expériences de chacune. Le recours aux termes "genre », " égalité » ou encore « discrimination » est réservé pour les rapports alternatifs et les publications des associations. Ainsi, dans le dernier rapport alternatif soumis en décembre 2010 par le groupe de travail sur le statut des femmes palestiniennes au comité CEDAW, Sidreb se définit comme une association défendant l' « égalité de genre 28 ».

La mise en œuvre des enseignements de ces rencontres internationales passe par un mode de réappropriation et de légitimation sur le terrain beaucoup plus complexe. En effet, on peut questionner le sens donné au « genre » puisque Sidreh ne se définit pas comme une association féministe. Les travaux menés ailleurs sur cette question ont mis en évidence combien le genre, en circulant, avait fait l'objet de redéfinitions dans les politiques de développement ${ }^{29}$, d'une neutralisation de sa dimension critique ${ }^{30}$, comme de sa dimension politique ${ }^{31}$, étant devenu pour les bailleurs et bon nombre d'associations synonyme de "femmes ", et entraînant ainsi une "neutralisation du féminisme ». Dans son étude des associations de femmes marocaines, Aurélie Dammame a justement souligné que ce qui est «bénéfique pour les femmes est alors conçu selon les schémas généraux des développeurs : alphabétisation et les activités génératrices de revenus 32 ». En outre, la notion même de genre n'induit pas forcément le féminisme. Comme le précisait Uma Narayan sur sa propre expérience : «Une femme n'est pas féministe simplement parce qu'elle est consciente des dynamiques de genre à l'œuvre dans sa famille et dans sa culture ", même si elle en a une conscience critique. Il se peut que les femmes aient une conscience de ces dynamiques mais les considèrent comme des problèmes personnels [...] ce qui permet aux femmes de devenir des féministes au plein sens du terme, c'est de développer des liens politiques avec les autres femmes et leurs expériences, d'analyser politiquement leurs problèmes et d'en chercher les solutions politiques 33 ».

28. Caulier M., "La conquête du "genre" et l'anthropologie au Mexique », Les rapports de sexe sont-ils solubles dans le genre?, Journal des anthropologues, n¹24-125, mai 2011, p. 67.

29. Verschuur C., "Quel genre ? Résistances et mésententes autour du mot "genre" dans le développement ", Les mots du développement. Trajectoires et pouvoirs, Revue Tiers Monde, 4, 2009, pp. 785-803.

30. Cirstocea I., " Du "genre" critique au "genre" neutre : effets de circulation ", in MarquèsPerreira B. et alii (eds.), Au-delà et en deçà de l'Etat. Le genre entre dynamiques transnationales et multiniveaux, Louvain-la-Neuve, Academia Bruylant, 2010, pp. 183-196.

31. Bisilliat J., "Le genre : une nécessité historique face à des contextes aporétiques», in Bisilliat J. (ed.), Regards de femmes sur la globalisation : approches critiques, Paris, Karthala, 2003, pp. 153-170.

32. Damamme A., «Usages du genre dans les projets de développement: L'exemple des associations marocaines " Les rapports de sexe sont-ils solubles dans le genre?, Journal des anthropologues, ${ }^{\circ} 124-125$, mai 2011, p. 103.

33. Narayan U., "Les cultures mises en questions "Occidentalisation", respect des cultures et 
Cependant, la polysémie du «genre » ne doit pas nous faire refuser son utilisation ${ }^{34}$, elle peut surtout nous obliger à considérer la manière dont cette notion circule, est comprise et surtout utilisée par les associations locales de femmes. Il est indéniable que ces dernières ne souscrivent pas à une remise en cause des identités de genre mais préfèrent lutter contre certaines inégalités hommes-femmes (éducation, emploi, santé) et surtout au projet d'émancipation individuelle. Dans des cadres sociétaux fortement marqués par les logiques familiales (voire tribales) et communautaires, où les divers canaux de médiation ont longtemps empêché toute existence sociale et politique individuelle des femmes, il n'est pas étonnant de constater le succès des programmes d'émancipation individuelle. Pour autant, cette autonomisation personnelle ne vise pas au bouleversement des cadres familiaux et communautaires qui restent des espaces sécurisants et considérés comme " authentiques » dans un contexte israélien où les Bédouins (et plus largement les Palestiniens) sont discriminés. En Israël, la plupart des Bédouines engagées dans l'associatif ne souhaitent pas « révolutionner » les normes sociales, les règles matrimoniales, la centralité de la famille, de la maternité ou encore le code d'honneur. La plupart continue de se marier avec des Bédouins, de préférence de leur famille élargie ${ }^{35}$, tout en inventant des canaux d'autonomisation par les études, le travail, ou encore l'engagement associatif.

Par ailleurs, certaines savent aussi que les questions de genre sont une rhétorique appréciée des bailleurs et surtout des autorités israéliennes car elles renforcent les stéréotypes dominants sur le «traditionnalisme » arabe, bédouin, musulman. En allant dans ce sens, elles savent qu'elles risquent d'être considérées comme des traîtres à leur communauté, à la cause arabe et palestinienne, et comme des relais de l'impérialisme occidental. C'est bien cette singularité du contexte local et national qui est importante pour comprendre la

féministes du tiers monde ", Genre, postcolonialisme et diversité des mouvements de femmes, Cahiers Genre et développement, n7, 2010, pp. 469-500.

34. Scott J.W, «Le genre : une catégorie d'analyse toujours utile ? ", Diogène, vol. 1, n²25, 2009, pp. 5-14.

35. Dans l'idéal, les Bédouins privilégient le mariage avec la cousine parallèle patrilatérale réelle ou classificatoire. Par extension, la pratique du Badal (échange) s'est aussi développée, permettant, par le mariage croisé de deux frères avec deux sœurs, d'annuler la dote et de souder les deux familles. Ce qui est recherché dans ces pratiques matrimoniales, c'est l'identité et l'équivalence entre les deux partenaires pour éviter les risques hiérarchiques qu'engendrerait un mariage hypergamique (épouse de rang social inférieur à son époux) ou hypogamique (épouse de rang social supérieur à son époux) (Kressel G., Descent through Males. An Anthropological Investigation into Patterns Underlying Social Hierarchy, Kinship, and Marriage among Former Bedouin in the Ramla-Lod Area (Israel), Mediterranean Language and Culture Monograph Series 8, Wiesbaden, Otto Harrassowitz, 1992). Les pratiques matrimoniales s'inscrivent dans un imaginaire de valeurs féminines et masculines qui font que les deux parties mettent en scène les rapports de pouvoir qui existent entre deux groupes. "Les époux respectifs représentant chacun leur groupe d'origine, le groupe prenant l'épouse est masculinisé tandis que le groupe donneur est féminisé » (Parizot C., Le mois de la bienvenue. Réappropriations de mécanismes électoraux et réajustements de rapports de pouvoir chez les Bédouins du Néguev (Israël), Thèse de Doctorat, Paris, EHESS, 2001, p. 53). Les pratiques matrimoniales sont cependant en train de changer, en particulier le Badal qui est de plus en plus critiqué par les jeunes au nom des risques médicaux de consanguinité. 
manipulation $\mathrm{du}$ " genre » et les stratégies d'adaptation mises en place par quelques militantes bédouines. Il s'agit ici de se départir de l'idée victimaire et naive des femmes du Sud accaparant sans les comprendre des notions préalablement vidées de leur sens subversif sous l'effet des circulations transnationales dans une mondialisation néolibérale.

\section{Les maux (mots) du féminisme}

Cette complexité s'observe très clairement dans le travail de l'association bédouine féministe $M a^{\prime}$ an, qui fonctionne comme une structure de coordination des différentes associations de femmes bédouines du Néguev. Créée en 1999, Ma'an est dirigée par Safa Shahadeh, la quarantaine, originaire de la région centrale du Triangle en Israël, donc non bédouine, féministe et militante communiste depuis toute jeune. Le profil de Safa est d'autant plus singulier qu'elle est musulmane non voilée, célibataire, laïque et militante politique. Elle se définit comme féministe et lutte depuis plusieurs années pour que les Bédouines affirment leur identité palestinienne. Ces deux luttes sont ainsi très connectées dans son discours. Son combat n'est pas chose aisée parmi les associations de femmes bédouines qui insistent, pour certaines, de plus en plus sur leur identité palestinienne ${ }^{36}$, mais refusent largement de se définir comme féministes. C'est d'ailleurs un constat qui a été fait plus largement pour l'ensemble des associations de femmes arabes palestiniennes en Israël, qui insistent toutes sur leur identité nationale mais préfèrent développer un discours social sur l' " aide aux femmes », plutôt que sur « la lutte et les droits des femmes 37 ». Il s'agit ici d'un refus de politisation des identités, soient-elles ethniques, nationales ou de genre. À la différence de quelques associations ouvertement féministes en Galilée ou en Cisjordanie, qui sont fortement marquées par leurs liens avec les mouvements politiques, les associations bédouines ont toujours préféré rester en marge du jeu politique local ou national ${ }^{38}$. Elles sont ainsi très souvent réticentes à toute forme d'action collective politique. Il est ainsi intéressant d'observer qu'elles refusent d'une même voix le féminisme comme la politique partisane, pas seulement parce que les féministes arabes sont souvent des militantes communistes et nationalistes, mais aussi parce qu'elles ne croient pas aux projets portés par ces mobilisations.

36. Cette affirmation d'une identité palestinienne par les associations de femmes a été observée notamment dans le nord en Galilée, en particulier dans les associations dirigées par des militantes politiques, communistes et nationalistes (Marteu E., Les associations de femmes arabes en Israël..., op. cit.). Cette palestinité fut plus tardivement assumée dans la population bédouine du Néguev, pour au moins trois raisons : (1) la force de l'identité bédouine, (2) la politique israélienne de fragmentation et de différenciation identitaire au sein des Arabes israéliens, notamment à l'égard des Bédouins du Néguev, (3) le développement plus tardif dans les années quatre-vingts de relais associatifs et partisans porteurs de projets identitaires politiques.

37. Marteu E., ibid.

38. Marteu E., «Compléments ou alternatives ? Les relations entre associations de femmes et partis arabes palestiniens en Israël », Le Mouvement social, n²31, 2010, p. 45-62. 
$M a^{\prime}$ an travaille donc essentiellement sur des programmes d'« empowerment » et d' " égalité » entre les hommes et les femmes, mais en utilisant de plus en plus les notions de «féminisme » et de "genre », ce dernier terme étant retranscrit tel quel en arabe jender (الدندر). Sur son site web, l'association précise qu'elle « étudie les théories et philosophies féministes en essayant de les appliquer aux contextes culturels et politiques du Néguev. Ma'an estime qu'il est important de comprendre et d'analyser l'hégémonie actuelle et les relations de pouvoir qui existent dans le but de promouvoir un changement positif. Les femmes arabes bédouines du Néguev sont doublement marginalisées : elles sont des femmes dans une société traditionnelle patriarcale et elles sont des Arabes dans un État juif 39 ». Il s'agit ainsi d'une des très rares associations bédouines à cibler très clairement le problème des inégalités hommes-femmes et à organiser des groupes mixtes de discussion sur des sujets comme le genre, les relations de pouvoir hommes-femmes, les droits des femmes, le féminisme et l'oppression des femmes. Il s'agit aussi d'une des très rares associations à concilier lutte de genre et lutte nationale en croisant très clairement les rapports de pouvoir à l'œuvre dans la population bédouine du Néguev.

Mes enquêtes menées auprès de $M a^{\prime}$ an depuis 2004 mettent en évidence une inclinaison féministe de plus en plus affichée de l'association, ainsi qu'une affirmation identitaire palestinienne assumée, essentiellement sous l'impulsion de sa directrice Safa Shahadeh. L'association organise ainsi des visites dans des villes palestiniennes comme Ramallah en Cisjordanie, où les jeunes Bédouines en profitent pour faire les magasins et baigner pour quelques heures dans une atmosphère exclusivement arabe palestinienne. L'objectif est d'activer chez elles un sentiment national et leur identité palestinienne. Si ce positionnement est apprécié du plus grand nombre, en revanche la posture féministe de Safa ne fait pas l'unanimité auprès des membres des autres associations de femmes bédouines du Néguev. À plusieurs reprises, j’ai entendu des critiques à son encontre, qu'il s'agisse de sa radicalité féministe, de son engagement communiste laï ou encore du fait qu'elle ne soit pas bédouine. Certaines lui reprochent de ne pas comprendre la singularité de la communauté bédouine, la fragilité des femmes et les dangers d'une lutte féministe qui serait préjudiciable pour l'organisation sociale des rapports hommes-femmes dans le Néguev. Personne ne conteste l'utilité des programmes d'action à destination des femmes, mais beaucoup reprochent à Safa d'être trop « brutale » dans sa critique du patriarcat, qui semblerait oublier que les hommes bédouins sont, eux-aussi, victimes dans une société israélienne discriminante.

L'association $M a^{\prime}$ 'an reste d'ailleurs très prudente dans ses modes d'action, en essayant d'intégrer les hommes bédouins dans ses activités, non seulement en organisant des groupes de discussion mixtes, mais aussi en invitant des sheikhs et des représentants religieux à des conférences. En novembre 
2001, Ma'an a organisé une conférence intitulée « La communauté arabe bédouine dans le Néguev : tribalisme bédouin et modernité », pour discuter des transformations des logiques tribales bédouines à l'aune des récentes transformations socioéconomiques et culturelles en Israël. Plusieurs personnalités locales se sont exprimées, dont un juge islamique et un sheikh du Mouvement islamique. Comme le souligne Sarab Abu Rabbia ${ }^{40}$, plutôt que d'attaquer frontalement le tribalisme, $M a^{\prime}$ an a préféré donner la parole à des représentants religieux qui se sont chargés de remettre en question les règles tribales au regard de l'Islam, notamment dans les pratiques matrimoniales. $\mathrm{Vu}$ l'importance prise par le Mouvement islamique dans la région depuis les années quatre-vingt-dix, en tant qu'acteur social, politique et spirituel, les associations de femmes restent très respectueuses de la religion ${ }^{41}$. Pour autant, la parole fut également donnée à une assistante sociale, qui expliqua les conséquences des contraintes matrimoniales et du code d'honneur sur les femmes. Le recours à différentes sources de légitimité, à la fois professionnelle et religieuse, leur a permis d'interroger les logiques tribales sans risquer d'être accusées de radicalité.

Ainsi, si les notions d' «empowerment » et de « genre » ne font l'objet d'aucun débat sur le terrain, beaucoup de femmes bénéficiaires ignorant complètement ces termes, le "féminisme » suscite des tensions et, en retour, es précautions. La distinction de plus en plus évidente entre niswiyya (féministe) et nisa'iyya (féminin) fait l'objet de positionnements clairs de la part des associations de femmes. Hormis $M a^{\prime}$ 'an, toutes les autres associations de femmes bédouines se définissent comme des associations de femmes (jama'iyyat an nisa) ou féminines (jama'iyyat nisa'iyya). Lors des entretiens, j'ai pu observer la réticence de la plupart des femmes pour l'usage du terme "féminisme ». Elles rejettent ce terme qu'elles pensent trop politique, trop radical, trop occidental, ou encore trop perverti. Certaines préfèrent le laisser pour caractériser les féministes juives occidentalisées, ou les féministes arabes du nord, c'est-àdire urbaines et souvent chrétiennes, militantes, communistes et laïques. L'usage du féminisme sert ainsi à marquer une frontière évidente, d'ordre tant politique, social qu'ethnique et confessionnel.

Ces critiques ne sont pas nouvelles, et ont été observées dans beaucoup d'autres contextes où le féminisme postcolonial s'est construit en opposition au féminisme dit occidental et libéral ${ }^{42}$. Certains mouvements de femmes

40. Abu Rabbia S., “The Activism of Bedouin Women: Social and Political Resistance”, $H A G A R$, vol. 7, n², 2007, pp. 67-84.

41. Il faut souligner ici que l'intégration de l'Islam dans les pratiques et discours des associations de femmes bédouines ne relève en aucune manière d'un "féminisme islamique », dont le récent succès reste lui-même à discuter. Voir sur ce sujet les publications dirigées par Stéphanie Latte Abdallah (dir.), Thema Le féminisme islamique, Critique internationale, n46, 2010 ; Dossier thématique Féminismes islamiques, Revue des mondes musulmans et de la Méditerranée, $\mathrm{n}^{\circ} 128,2010$.

42. Mohanty C.T., "Under Western Eyes: Feminist Scholarship and Colonial Discourses", 
développés dans cette vaine critique et autonomiste ont pu aller jusqu'au refus même du label «féminisme ». Il ne s'agit pas simplement d'une neutralisation induite par les bailleurs de fonds internationaux, qui, en dépolitisant le " genre », aurait également neutralisé le féminisme. Sur le terrain, la réalité est beaucoup plus complexe. Le refus du label «féminisme » trouve différentes explications : (1) le refus d'une idéologie inadaptée car considérée comme radicale, inappropriée et importée ; (2) le refus d'un cadre de pensée considéré comme dangereux parce que porteur de remise en cause des cadres sociaux de genre et des identités sexuées ; (3) le refus d'une action collective pouvant noyer et rendre inaudible la diversité des opinions et des subjectivités féminines ; (4) le refus pédagogique du label féministe (parce que trop controversé) tout en élaborant des stratégies de conscientisation et de transformations des rapports de genre.

Par ailleurs, on peut retrouver en filigrane un débat bien connu dans le féminisme postcolonial sur la délicate articulation (voir hiérarchisation) entre lutte de genre et lutte nationale, ethnique et anti-coloniale ${ }^{43}$. Ce débat est d'ailleurs prégnant dans les modes d'action des associations de femmes palestiniennes, en Israël 44 et dans les Territoires occupés ${ }^{45}$, où elles sont prises entre différents enjeux de lutte. La mobilisation féminine bédouine dans le Néguev se trouve aujourd'hui, elle aussi, au centre de ce dilemme, d'autant plus que les Bédouins clament leur identité palestinienne justement par l'intermédiaire des associations de femmes, de droits de l'homme ou de développement. Les relations entre féminismes et nationalismes sont donc d'autant plus complexifiées que les Bédouins renégocient leurs rapports avec l'État israélien, non plus seulement en tant que communauté bédouine, mais aussi en tant que minorité palestinienne. Or, les Bédouins ont longtemps été considérés par les Israéliens comme une minorité ethnique qu'il fallait gérer indépendamment des autres citoyens arabes. L'échec de cette tentative de fragmenta-

Feminist Review, n³0, Autumn, 1988, p. 61-88. Voir également les travaux du Black Feminism (Dorlin E. (ed.), Black Feminism, Anthologie du féminisme africain-américain, 1975-2000, Paris, L'Harmattan, 2007) ou encore les mobilisations féministes développées dans d'autres contextes en Asie et en Amérique Latine (voir entre autres Verschuur C. (ed.), "Genre, postcolonialisme et diversité des mouvements de femmes ", Cahiers Genre et développement, $\left.\mathrm{n}^{\circ} 7,2010\right)$.

43. Voir entre autres Jayawardena K., Feminism and Nationalism in the Third World, London, Zed, 1986 ; McClintock A., Mufti A., Shohat E. (eds), Dangerous Liaisons. Gender, Nation and Postcolonial Perspectives, Minneapolis, University of Minnesota Press, 1997 ; Yuval Davis N., Gender and Nation, London, Sage Publications, 1997 ; Mayer T. (ed.), Gender Ironies of Nationalism. Sexing the Nation, London, Routledge, 2000.

44. Marteu E., Les associations de femmes arabes en Israël..., op. cit. ; Abdo J., Les associations de femmes et féministes palestiniennes dans les frontières de 1948, Mada al Carmel, 2008 (en arabe).

45. Voir entre autres Abdo N., "Nationalism and Feminism: Palestinian Women and the Intifada - No Going Back?”, in Moghadam V., Gender and National Identity: Women and Politics in Muslim Societies, London and Atlantic Highlands, Zed Books, Karachi, Oxford University Press, pp. 148-170. Jad I., “Claming Feminism, Claiming Nationalism: Women’s Activism in the Occupied Territories”, in Basu A. (ed.), The Challenge of Local Feminism, Women's Movements in Global Perspective, Boulder, Westview Press, pp. 226-248. 
tion interne 46 a mis en évidence les risques politiques de leur non développement socioéconomique, à l'heure où ils articulent leurs griefs à la lutte palestinienne, voire à la lutte islamique. Ce faisant, l'aide à destination des femmes bédouines ne relève plus seulement d'une lutte contre la pauvreté, elle est aussi clairement un moyen de développer, de pacifier et de sécuriser la région avec l'aide d'agents réformateurs. Les associations de femmes sont ainsi au coeur de cette recomposition de la militance palestinienne en Israël, considérées par les uns comme des agents de démocratisation et de pacification, et par les autres comme des actrices de la lutte nationale palestinienne, y compris en Israël. Ainsi, au-delà des relations hommes-femmes, l'usage du « genre », de l' « empowerment » et du «féminisme » a une résonance politique inscrite au cour du conflit israélo-palestinien et de la complexité du positionnement des Arabes palestiniens en Israël.

La circulation transnationale des idées ${ }^{47}$, des mots et des pratiques induit indéniablement des processus de reconfiguration et de reconstruction des significations et des subjectivités. Les acteurs internationaux (ONU, ONG, bailleurs de fonds) ont une responsabilité dans la diffusion de politiques de genre considérées comme "féministement» neutralisées. Pour autant, les associations locales de femmes ne sont pas de simples structures d'intériorisation et d'enregistrement des injonctions internationales. Elles sont aussi des actrices qui " s'expriment 48 », conscientes et informées, qui s'accommodent d'autant mieux de différents répertoires d'action qu'elles-mêmes sont prises dans des rapports de pouvoir multi-niveaux et des contraintes locales et nationales. Enfin, force est de reconnaître que ces dirigeantes et membres associatives ne souhaitent pas forcément transformer leurs capacités d'action individuelles en action collective politique. Deux limites se posent ici : celle de la mise en mouvement, et celle de la sororité. Jusqu'où veulent-elles et peuventelles aller dans la solidarité et la mise en mouvement des femmes ? Dans un contexte fragmenté, communautaire et conflictuel, comme le contexte israélopalestinien, il n'est pas certain que les entreprises féminines aient les moyens et les envies de subvertir les frontières de genre comme les frontières ethniques et confessionnelles.

46. Yonah Y., Abu-Saad I., Kaplan A., "De-Arabization of the Bedouin: A Study of an Inevitable Failure”, in Kemp A., Newman D., Ram U., Yiftachel O., Israelis in Conflict: Hegemonies, Identities and Challenges, Brighton, Portland, Sussex Academic Press, 2004, pp. 65-80.

47. Pour reprendre les termes de Bourdieu P., "Les conditions de la circulation internationale des idées ", Actes de la recherche en sciences sociales, 145, 2002, pp. 3-8.

48. Pour répondre à la question posée par Spivak G.C., Les subalternes peuvent-elles parler?, Editions Amsterdam, 2006 [1988]. 